\title{
Dysphonia as First Symptom of Late-Onset Myasthenia Gravis
}

\author{
Manuel Montero-Odasso, MD, $P h D^{1,2,3}$ \\ ${ }^{1}$ McGill Division of Geriatric Medicine, McGill University, Montréal, QC, Canada; ${ }^{2}$ Geriatric Assessment Unit (EFA) and Geriatric Medicine \\ Program, Hospital Italiano de Buenos Aires, Buenos Aires, Argentina; ${ }^{3}$ Solidage: McGill University, Université de Montréal Research Group \\ on Integrated Services for Older Persons, Montréal, QC, Canada.
}

\begin{abstract}
Myasthenia gravis (MG) is an autoimmune disorder of the neuromuscular junction that causes muscle weakness and fatigue. Fluctuating fatigue of skeletal muscles is the key clinical feature. Late-onset MG is more frequent in elderly men and is often misdiagnosed. While involvement of oropharyngeal musculature has been described with symptoms of dysphagia and slurred speech, the presence of fluctuating dysphonia as the first symptom of late-onset MG has not been emphasized. The case of an elderly man, who demonstrated voice changes and later swallowing impairment with weight loss, is reported. This case presentation of late-onset MG emphasizes that this form of the disease should be considered in the differential diagnosis of acute onset dysphonia in elderly persons.
\end{abstract}

KEY WORDS: myasthenia gravis; dysphonia; dysphagia; late-onset myasthenia; aged.

DOI: $10.1111 /$ j.1525-1497.2006.00343.x

J GEN INTERN MED 2006; 21:C4-C6.

$\mathrm{M}$ yasthenia gravis (MG) is an autoimmune disease mediated by immunoglobulin G (IgG) anti-acetylcholine receptor antibodies and a subsequent loss of postsynaptic receptors. ${ }^{1}$ The prevalence varies from 5 to 15 cases per 100,000 , whereas the incidence is about 1 case per 100,000 in the general population. ${ }^{2-4}$ Almost one third of all cases present in the elderly population. In men, the highest incidence is among those older than 50 years, with a peak age around 70 . Women have 2 incidence peaks: 1 between the ages 20 and 40 years and 1 at approximately 70 years of age. ${ }^{4}$

Although fatigability of peripheral skeletal muscle is the hallmark of the disease, it can be absent in the bulbar forms. In particular, the isolated bulbar presentation in late-onset MG is not rare and may be confused with diseases of the oropharynx and neurologic conditions. ${ }^{5-7}$ Dysphonia and dysphagia, as manifestations of this form, occur because of the pathologic fatigability of striated muscles in the pharynx and esophagus. ${ }^{8}$ The case of an elderly man with fluctuating dysphonia, and later swallowing impairment and weight loss, is described.

\section{CASE REPORT}

A 77-year-old man consulted his primary care physician complaining of an acute onset of voice tone changes and slurred

The authors have no conflicts of interest to declare for this article.

Address correspondence and requests for reprint to Dr. MonteroOdasso: Division of Geriatric Medicine, Jewish General Hospital, McGill University, Lady Davis Institute and Solidage Research Group, 3755 Cote-Ste-Catherine, Montréal, QC, Canada, H3T $1 E 2$ (e-mail: manuel. montero@hospitalitaliano.org.ar). speech. He described a definite change in the quality of his voice and disturbance of his speech fluency. Initially, no choking or coughing was reported and all the symptoms resolved completely by the next morning. His past medical history was unremarkable except for uncontrolled hypertension and overweight. He was cognitively intact and independent in both basic and instrumental activities of daily living.

For a possible transient ischemic attack, he was referred to a neurologist. The computer tomography (CT) scan of the brain was normal, as was a Doppler ultrasound exam of the neck vessels. His blood pressure was brought under strict control with enalapril-hydrochlothiazide $20 / 25 \mathrm{mg}$ /day, and he was started on aspirin $325 \mathrm{mg} /$ day. The dysphonia and speech symptoms recurred almost every night but with full recovery by the following morning. No other associated symptoms such as diplopia, parasthesias, headaches, respiratory problems, or limb weakness were described. As the voice changes presented in every episode, he was referred for an otolaryngology assessment. The only abnormality found was slightly congested vocal cords, and a speech therapist assessment was normal. On subsequent weeks, he started to experience difficulty swallowing toward the end of the meal. The swallowing impairment persisted and progressed, leading him to begin to skip his evening meal. Further blood chemistry and an upper gastrointestinal endoscopy were normal.

After 5 weeks of symptoms and a $5 \mathrm{~kg}$ weight loss, he was referred to a geriatrician for assessment. As the diagnosis of late-onset MG was suspected, a blood workup was performed and a trial treatment with pyridostigmine was offered. This proved positive, with a definite but transient improvement in his speech volume, voice clarity, and swallowing function. Blood tests revealed an acetylcholine receptor antibody test (AchR-abs) positive at $2.8 \mathrm{mmol} / \mathrm{L}$ (normal range 0.0 to 0.25 ). The diagnosis of late-onset bulbar MG was confirmed and he was started on oral pyridostigmine $60 \mathrm{mg} 4$ times a day, increasing to 6 times a day on review 2 weeks later. At follow-up 1 month later, while significant improvement had occurred, there was still evidence of slight swallowing problems, again, in the evening. As a result, it was decided to increase the dose of pyridostigmine and to add $20 \mathrm{mg} /$ day of prednisone, increasing gradually to $60 \mathrm{mg}$ daily. His symptoms improved and at the 3-month follow-up, he was completely asymptomatic. The pyridostigmine was gradually reduced over 1 month but the prednisone was maintained for the following 4 months and

Manuscript received August 29, 2005

Initial editorial decision October 18, 2005

Final acceptance November 3, 2005 
tapered slowly for 2 months to the minimal effective dose for this patient, which was $10 \mathrm{mg} /$ day.

As a complication of the steroid therapy, the patient developed unstable hypertension and hyperglycemia, which resolved after reducing the dose. He remained asymptomatic at his 8-month follow-up. Neurologic examination was entirely normal, although his AchR-abs remained positive at $2.00 \mathrm{mmol} / \mathrm{L}$. Computed tomography of the mediastinum did not show any thymic abnormality.

\section{DISCUSSION}

This case portrays a not uncommon presentation of late-onset MG in an elderly man. As described previously, the misdiagnosis of a transient ischemic attack was made at the initial presentation. ${ }^{6}$ Slurred and nasal speech has been described in patients with MG to be related to tongue and palate muscle weakness; however, dysphonia as an initial presentation has not been emphasized previously in late-onset MG as it has been in the early-onset form. ${ }^{9,10}$ Although this patient developed other associated symptoms, dysphonia was the single manifestation of MG in the first weeks. As elderly persons who suffer from acute dysphonia are more likely to have a disease progress rather than age-associated changes, late-onset MG should be considered in the differential diagnosis. ${ }^{11}$ Common causes of dysphonia and laryngeal muscle weakness in elderly persons are summarized in Table 1.

Myasthenia gravis is a masquerader in elderly persons, as the fluctuating weakness, a cardinal feature for diagnosis, can be mistaken for age-associated changes or another acute comorbid condition. While MG most commonly occurs in younger people, almost a third of all patients are seen in the seventh decade of life or beyond, with a preponderance of men. ${ }^{2,3}$ Onset of MG in the older population is increasingly being recognized and is designated as "late-onset MG." ",,9 In the isolated bulbar form, as in this case, patients may report alterations of the voice (e.g., softness, breathiness, and hoarseness), slurred or nasal speech, and difficulty chewing or swallowing. ${ }^{9,10}$ Limb weakness is a rare initial complaint, occurring in $14 \%$ to $27 \%$ of cases, and should be differentiated from nonspecific generalized fatigue. ${ }^{9}$ Similar to other forms of myasthenia, the symptoms are less obvious upon awakening or after rest and become progressively worse later in the day, as demonstrated by this case. Acetylcholine receptor antibody test serum levels are the most specific diagnostic test for MG; however, the titers can be lower or undetectable in older patients. ${ }^{4}$ In these seronegative patients, muscular tyrosine kynase antibodies (MuSK

Table 1. Common Causes of Dysphonia in Elderly Persons*

\begin{tabular}{ll}
\hline \hline Category & \multicolumn{1}{c}{ Common Causes } \\
\hline $\begin{array}{c}\text { Central neurologic } \\
\text { disorders } \\
\text { Local neurologic } \\
\text { disorders. }\end{array}$ & Stroke, Parkinson's disease, Alzheimer's disease \\
$\begin{array}{l}\text { Benign vocal fold } \\
\text { lesions }\end{array}$ & $\begin{array}{l}\text { Reinke's edema, benign and dysplastic } \\
\text { epithelial lesions }\end{array}$ \\
$\begin{array}{l}\text { Inflammatory } \\
\text { disorders }\end{array}$ & $\begin{array}{l}\text { Laryngitis sicca, medication effect, } \\
\text { laryngopharyngeal reflux }\end{array}$ \\
Neoplasias & Laryngeal epidermic cancer \\
\hline *Based on data from Woo et al. ${ }^{11}$ \\
$\begin{array}{l}\text { 'Presbylarynges is not a common disorder and should be a diagnosis of } \\
\text { exclusion. }\end{array}$
\end{tabular}

Table 2. Difference Between Early Onset and Late-Onset MG*

\begin{tabular}{|c|c|c|}
\hline Characteristics & Early-Onset MG & Late Onset MG \\
\hline Age peak (y) & 30 & 65 \\
\hline Female:men ratio & 4:1 & $1: 3$ \\
\hline $\begin{array}{l}\text { Proportion of total } \\
\text { MG cases }\end{array}$ & $65 \%$ to $70 \%$ & Up to $30 \%$ \\
\hline $\begin{array}{l}\text { Presenting } \\
\text { symptoms }\end{array}$ & $\begin{array}{l}\text { Weakness and fatigue } \\
\text { are more common } \\
\text { Ocular and extremity } \\
\text { signs frequently } \\
\text { presented }\end{array}$ & $\begin{array}{l}\text { Peripheral weakness } \\
\text { and fatigue can be absent } \\
\text { Ocular signs less common } \\
\text { bulbar signs are more } \\
\text { common }\end{array}$ \\
\hline Thymoma presence & Rare & More common \\
\hline $\begin{array}{l}\text { Acetylcholine } \\
\text { receptor antibody } \\
\text { test (AchR-abs) }\end{array}$ & Present in up to $90 \%$ & $\begin{array}{l}\text { Absent in up } 40 \% \\
\text { (seronegative MG) }\end{array}$ \\
\hline HLA associations & HLA-DR3 & None \\
\hline
\end{tabular}

Ocular signs include ptosis, gaze pareses and diplopia. Bulbar signs include dysphagia, dysphonia, tongue weakness, slurred speech, and chewing problems.

${ }^{*}$ From Slesak et al. ${ }^{4}$ Aarli et al. ${ }^{5}$ and Sharp et al. ${ }^{9}$

MG, myasthenia gravis.

abs.) have been described as an alternative test. A positive response to edrophonium chloride (Tensilon ${ }^{\circledR}$ test) can confirm the diagnosis but should be implemented with caution in elderly persons because of side effects. In particular, in patients with oropharyngeal weakness and difficulty handling their oral secretions, Tensilon ${ }^{\circledR}$ test may increase these secretions because of its muscarinic cholinergic effects. ${ }^{12}$ Corroborative electrophysiologic changes in repetitive stimulation tests may also be helpful in establishing the final diagnosis.

Late-onset MG may differ from the classic form in several ways as is described in Table $2 .{ }^{5}$ It is more severe and less likely to remit, with the involvement of the bulbar musculature being more common. ${ }^{5,9}$ Response to conventional treatment can be less satisfactory than in younger patients. Thymomas are much more common in the late-onset form and because of its associated morbidity and mortality they should be carefully screened for in this population. ${ }^{5}$ Finally, special attention should be paid when corticosteroid therapy is initiated in elderly persons because of their vulnerability to secondary effects and the possible precipitation of a myasthenic crisis.

Because of confusion with signs of the aging process or from age-related comorbidities, it has been suggested that MG might be underdiagnosed or misdiagnosed in older people. ${ }^{13}$ When faced with new-onset dysphonia and isolated difficulties of speech in an older person, clinicians should consider MG as a diagnostic possibility, especially in the presence of fluctuating symptoms. ${ }^{7,9}$ A high index of clinical suspicion and a careful history are required for making the diagnosis.

In conclusion, this case report draws attention to the fact that dysphonia can be the first and single symptom of the isolated bulbar presentation of late-onset MG. Timely consideration of MG in evaluating this symptom is crucial to prevent complications and to improve the quality of life of these elderly patients.

\section{KEY POINTS}

- Late-onset MG may mimic otolaryngology diseases because of its frequent bulbar presentation.

- Delay in diagnosis is common because of lack of recognition and misdiagnosis. 
- Acetylcholine receptor antibody test is considered as the gold standard for diagnosis; however, older adults can be seronegative as this test is not a perfect reference standard.

- Myasthenia gravis should be considered in the differential diagnosis of acute onset dysphonia in elderly people, particularly men.

I am indebted to A. Mark Clarfield, MD (Departments of Geriatrics at Ben Gurion and McGill Universities), and Gillian Fisher, $M D$ (Division of Geriatric Medicine at McGill University), for their thoughtful and critical review of the manuscript. I am grateful to Jack Gaiptman, MD (Maimonides Geriatric Center at McGill University), for his kind help in the manuscript corrections. Dr. Montero Odasso holds a clinical and research fellowship award from Maimonides Geriatric Centre and from the Division of Geriatric Medicine, McGill University, Montréal, Canada. This case was presented at the Inter-Hospital Ground Rounds, Internal Medicine Service, Hospital Italiano de Buenos Aires, Buenos Aires, Argentina in September 2001.

\section{REFERENCES}

1. Drachman DB. Myasthenia gravis. N Engl J Med. 1994;330:1797-810.
2. Robertson NP, Deans J, Compston DA. Myasthenia gravis: a population based epidemiological study in Cambridgeshire, England. J Neurol Neurosurg Psychiatry. 1998;65:492-6.

3. Schon F, Drayson M, Thompson RA. Myasthenia gravis and elderly people. Age Ageing. 1996;25:56-8.

4. Slesak G, Melms A, Gerneth F, et al. Late-onset myasthenia gravis: follow-up of 113 patients diagnosed after age 60. Ann NY Acad Sci. 1998;841:777-80.

5. Aarli JA, Romi F, Skeie GO, Gilhus NE. Myasthenia gravis in individuals over 40. Ann NY Acad Sci. 2003;998:424-31.

6. Ngeh JKT, McElligott G. Myasthenia gravis: an elusive diagnosis in older people. J Am Geriatr Soc. 2001;49:683-4.

7. Kleiner-Fisman G, Kott HS. Myasthenia gravis mimicking stroke in elderly patients. Mayo Clin Proc. 1998;73:1077-8.

8. Chua E, McLoughlin C, Sharma A. Myasthenia gravis and recurrent falls in an elderly patient. Age Ageing. 2000;29:83-4.

9. Sharp HR, Degrip A, Mitchell DB, Heller A. Bulbar presentations of myasthenia gravis in the elderly patient. J Laryngol Otol. 2001;115:1-3.

10. Chang CH, Lee KW, Kuo WR. Dysphonia as the initial symptom of myasthenia gravis. J Otolaryngol. 2004;33:57-9.

11. Woo P, Casper J, Colton R, Brewer D. Dysphonia in the aging: physiology versus disease. Laryngoscope. 1992;102:139-44.

12. Pascuzzi RM. The edrophonium test. Semin Neurol. 2003;23:83-8.

13. Vincent A, Clover L, Buckley C, et al. UK myasthenia gravis survey. Evidence of underdiagnosis of myasthenia gravis in older people. $\mathrm{J}$ Neurol Neurosurg Psychiatry. 2003;74:1105-8. 